\title{
Mass Effect from Hepatomegaly in Polycystic Kidney Disease
}

\author{
Anil Chalisey, MRCP and Mahzuz Karim, PhD FRCP \\ Department of Renal Medicine, Norfolk and Norwich University Hospital, Norwich, Norfolk, UK.
}

KEY WORDS: adult polycystic kidney disease; polycystic liver; mass effect.

J Gen Intern Med 29(12):1713

DOI: $10.1007 / \mathrm{s} 11606-014-2963-1$

(c) Society of General Internal Medicine 2014
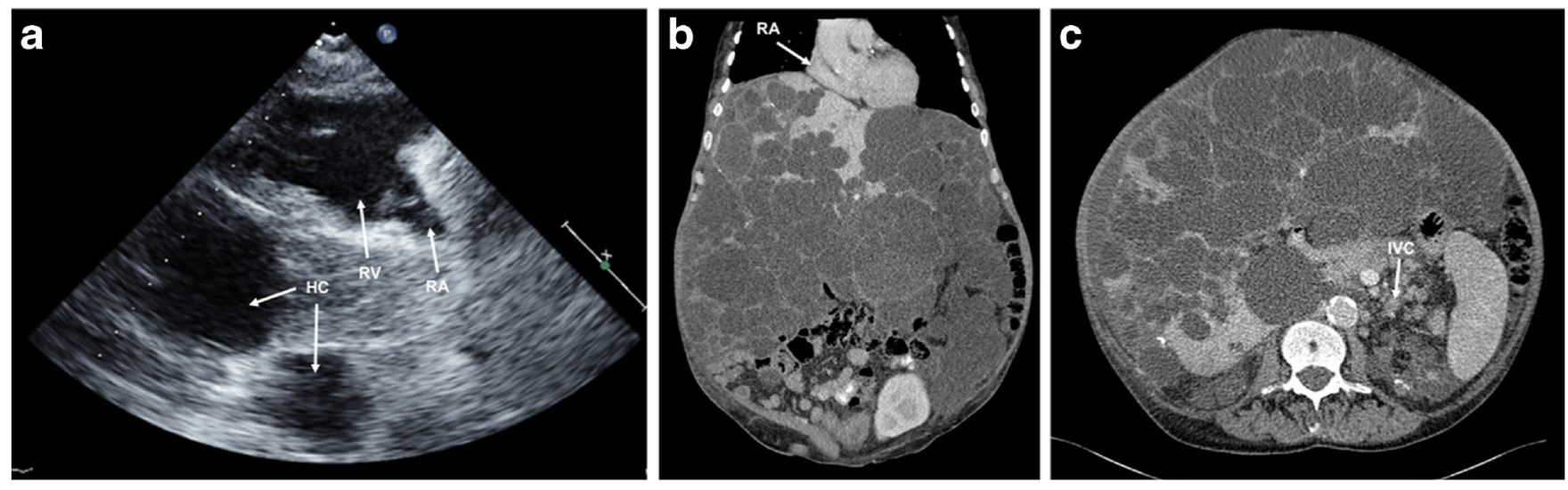

Figure 1

\begin{abstract}
A 66-year-old man presented with progressive lower limb edema and an elevated jugular venous pressure. He had undergone renal transplantation 14 years earlier for adult polycystic kidney disease. Serum creatinine and liver function tests were normal. Echocardiogram (Fig. 1a, online video) showed normal left ventricular function (ejection fraction $68 \%$ but compression of the right atrium (RA) adjacent to the right ventricle (RV) by hepatic cysts (HC). Computed tomography (CT) scan confirmed compression of the right atrium (Fig. 1b) and inferior vena cava (IVC) by an enlarged liver with numerous cysts (Fig. 1c). Hepatic transplantation was considered, but the patient's clinical condition deteriorated rapidly and he died before this could be pursued.

Hepatic involvement in polycystic kidney disease is common and can cause significant mass effect, compressing structures such as the great veins (leading to impaired venous drainage) and abdominal viscera
\end{abstract}

Electronic supplementary material The online version of this article (doi:10.1007/s11606-014-2963-1) contains supplementary material, which is available to authorized users.

Received March 29, 2014

Revised May 9, 2014

Accepted July 2, 2014

Published online August 16, 2014 (causing anorexia and weight loss, referred to as "lethal exhaustion" in severe cases). ${ }^{1,2}$ These symptoms may be severe enough to warrant percutaneous or surgical cyst drainage. Rarely, even in the absence of biochemical liver failure or portal hypertension, hepatic transplantation may be considered if the symptoms are sufficiently extreme to justify the associated risks of this surgery. ${ }^{1,3}$

Conflict of Interest: The authors declare that they do not have a conflict of interest.

Corresponding Author: Mahzuz Karim, PhD FRCP; Department of Renal Medicine, Norfolk and Norwich University Hospital, Norwich, Norfolk NR4 7UY, UK (e-mail: mkarim@doctors.org.uk).

\section{REFERENCES}

1. Schnelldorfer T, Torres VE, Zakaria S, Rosen CB, Nagorney DM. Polycystic liver disease: a critical appraisal of hepatic resection, cyst fenestration, and liver transplantation. Ann Surg. 2009;250:112-8.

2. Starzl TE, Reyes J, Tzakis A, Mieles L, Todo S, Gordon R. Liver transplantation for polycystic liver disease. Arch Surg. 1990;125:575-7.

3. Krohn PS, Hillingso JG, Kirkegaard P. Liver transplantation in polycystic liver disease: a relevant treatment modality for adults? Scand $\mathrm{J}$ Gastroenterol. 2008;43:89-94. 